\title{
BS09
}

\section{Land Broadband Acquisition: Where do We Stand on the Receiver Side?}

J.J.P. Postel* (Sercel)

\section{SUMMARY}

Acquiring high quality broadband data in land seismic can be achieved thanks to a smart combination of the 4 following topics:

1. $\quad$ Adapted 3D design with high density.

$2 . \quad$ Single source.

3. Single sensor.

4. Adapted processing able to cope with enormous quantity of very noisy data.

This paper will focus on the third topic, trying to summarize the current status and the road ahead. 


\section{Introduction}

Designing high density wide azimuth land 3D surveys has always been the dream of the 3D seismic land designer. High channel count recording systems able to record several hundred thousand channels per recording point are now available to make this dream a reality.

For Vibroseis, recent technical evolutions on both the software and hardware side, have been largely addressed with new vibrator designs, sweeping low and high dwell sweeps. This leads to large data quality improvements on both sides of the spectrum. Most Middle East crews are now using single vibrators and simultaneous high productivity methodologies (slip-sweep, DS3, DS4, ISS...) with more than 10000 recorded VP per day. This has made cost effective high density 3D surveys accessible.

Thanks to the recording of very dense data (several million traces per $\mathrm{km}^{2}$ ), where surface noise is no longer aliased, the processing side has also evolved recently with new 3D algorithms enabling the drastic reduction of all kinds of noises (random, harmonic, blended, industrial, surface waves...). These algorithms are applied pre-stack after sorting the data in different modes (shot, receiver, crossspread, OVT, bin), then new migration schemes adapted to dense broadband data also help drastically to improve imaging (RTM, FWI ...). The efficient and rapid handling of terabytes of data recorded every day is more challenging but solutions with high performance computing using multi-core processors are currently being implemented. They are derived from technologies already mature in the "Big Data" businesses which already store and process petabytes of data.

\section{The receiver side}

The receiver side is currently the least mature of the 4 topics. Most land crews are still using strings of standard analog geophones. Unlike marine seismic acquisition where the group interval of $12.5 \mathrm{~m}$ is a quasi-standard of the industry, the distance between recorded channels in land can still vary from 5 to $75 \mathrm{~m}$ depending on the country, the client and evidently the target depth. At each digital channel, a group of analog sensors is deployed in an array designed to act as an anti-alias filter for sourcegenerated noises such as ground-roll. In the Middle East, these are frequently aerial arrays to help attenuate scattered noises. The number of geophones per A/D converter has been steadily decreasing (by factors of 3 to 10 fold in 10 years) but the deployment remains a manual operation requiring heavy logistics, manpower and thus high cost and HSE exposure. Currently in most high channel count crews in the Middle East, the group interval is $25 \mathrm{~m}$ with strings of 6 to 9 phones.

The number of crews using single sensors only represents a very few percent of the total active crews worldwide and among these crews, only a small portion use digital MEMS phones. It is true that using single sensor with group interval larger than $20 \mathrm{~m}$ put the data at risk of being aliased, but progress in processing algorithms have shown the advantage of digital vs analog filtering enabling to relax the minimum sensor interval when digitizing each sensor. Let us try to analyze the reasons why this technology has not been, so far, a commercial success and detail what is available today with a new generation of digital sensors. We will also propose what we think could help to accelerate their wider deployment.

\section{MEMS}

After 50 years of incremental enhancements of the conventional (electromagnetic) geophone, MEMS (micro-electro-mechanical-system) technology, similar to that used for instance in car airbag control circuits, smartphones and ink jet printers, were introduced for geophysical applications at the start of this millennium (Maxwell 2001). The new sensor design has low intrinsic noise and ultralow distortion and allows mass production of very closely matched components. A digital feedback system keeps a reference mass steady during recording, and the output is proportional to the correction force needed to keep the mass stationary when the ground is accelerated. This output is 
digital. Although they were introduced more than fifteen years ago for geophysical exploration, MEMS geophysical sensors only represent today a very few percent of the total number of sensors sold every year in the world.

MEMS sensors present many advantages. In the acceleration domain, their response is flat in amplitude and constant in phase from zero frequency (DC). They can actually measure the gravity field and thereby evaluate tilt angles relative to the vertical. In this technology, the output signal is a byproduct of the feedback loop and the elimination of a coil makes it insensitive to electro-magnetic noise. The weakness of the MEMS sensor is its higher noise in the low frequencies. The noise floor is the conversion of thermal noise into acceleration or into velocity. At any given frequency, any signal higher than the noise floor will be seen on an individual record while signal lower than the noise floor will be buried into the noise. The crossing point comparing to standard geophones was around $50 \mathrm{~Hz}$ in previous generations, but it is now below $10 \mathrm{~Hz}$ with the new one.

10 years ago, an article by Denis Mougenot on MEMS concluded:

There are 2 limitations to the use of MEMS sensors:

- One is geophysical (digital sensors are recorded as single sensors)

- The other is economic (manufacturing costs of MEMS compared to coil geophones)

Today's latest generation of MEMS (Lainé 2014) with high sensitivity, light weight, low power consumption and very low noise floor has now surpassed the technical drawbacks when compared to conventional geophones. Even at very low frequency $(<3 \mathrm{~Hz})$, where the noise floor can still be higher than the industrial or ambient noise in very quiet environments, thanks to the high density of single sensor, this noise is largely oversampled in the very low frequency and thus totally attenuated on high fold stack. The figure below compares signal and noise levels with theoretical values on an explosive record.

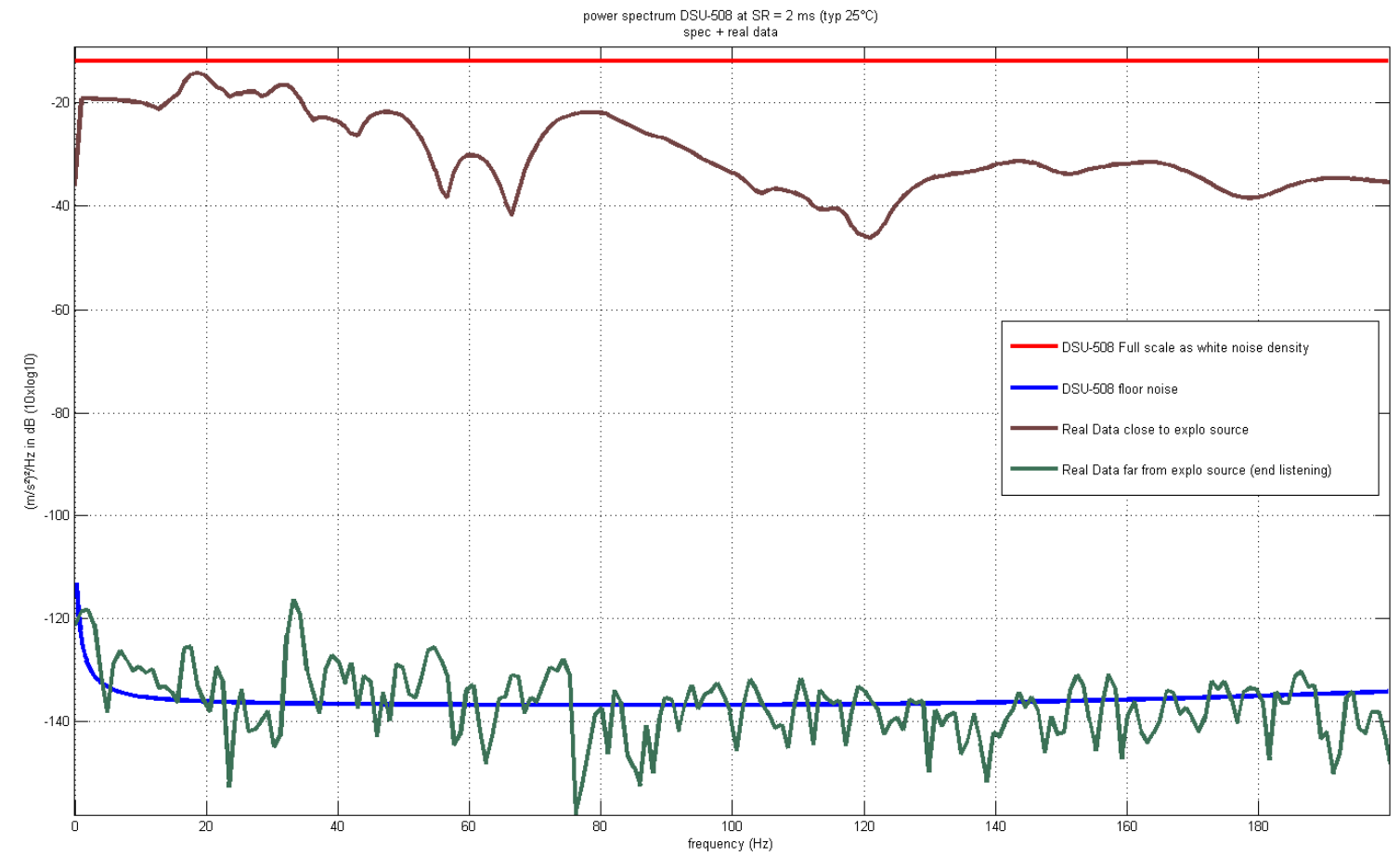




\section{Conclusion}

The last blocking factor is thus economic. When analyzing the cost of seismic sensors, it is necessary to look at the big picture and separate between the capital expenditure of the equipment (capex) and the operational expenses (opex) to transport, deploy, retrieve and maintain such equipment.

Reducing the cost in both of these areas are current ongoing processes:

- The cost of building this equipment is steadily reducing with mass production.

- The cost of the deployment logistic is also reduced with a streamlining of the field process.

It has to be noted that deploying single sensors with small group intervals reduces the total equipment weight to be transported as well as the number of personnel for deployment and retrieval, thus reducing overall operating expenses and the HSE exposure on the crew.

Another avenue on the capex and opex side, as in marine seismic streamer acquisition, will come with a standardization of the group interval. This will drastically simplify equipment manufacturers and contractors' equipment inventory and accelerate their global worldwide deployment.

\section{References}

Maxwell, P. et al [2001] Design through to production of a MEMS digital accelerometer for seismic acquisition. First Break, 19, 141-144

Mougenot, D. and Thorburn, N. [2004] MEMS-based 3C accelerometers for land seismic acquisition: is it time? The Leading Edge, 23 (3), 246-250

Lainé, J. and Mougenot, D. [2014] A high-sensitivity MEMS-based accelerometer. The Leading Edge, 33 (11), 1234-1242 